\section{Malária no Estado do Amapá, Brasil, de 1970 a 2003: trajetória e controle}

\author{
Malaria in Amapá State, Brazil, 1970-2003: \\ background and control
}

\author{
1 Secretaria de Estado \\ da Saúde do Amapá, \\ Macapá, Brasil. \\ 2 Instituto Macapaense \\ de Ensino Superior, \\ Macapá, Brasil. \\ ${ }^{3}$ Departamento de Medicina \\ Preventiva, Universidade \\ Federal de São Paulo \\ São Paulo, Brasil. \\ ${ }^{4}$ Centro de Desenvolvimento \\ do Ensino Superior em \\ Saúde, Universidade Federal \\ de São Paulo, São Paulo, \\ Brasil. \\ Correspondência \\ P. Goldenbers \\ Departamento de Medicina \\ Preventiva, Universidade \\ Federal de São Paulo. \\ Rua São Giusto 99, \\ São Paulo, SP \\ 04030-030, Brasil. \\ paulete@medprev.epm.br \\ pauletegolden@hotmail.com
}

\begin{abstract}
The present study aims to describe malaria occurrence from 1970 to 2003 in the State of Amapá, Brazil. With positive films obtained from health agencies, temporal series on the annual parasite incidence - API (per 1,000 inhabitants) were elaborated, taking into account population data available from the Brazilian Institute of Geography and Statistics. The results showed an increase in API values, although nonlinear, related to the rapid population growth associated with regional economic and social development during the period, ranging from $15.1 \%$ in 1970 to $74.0 \%$ o in 2000. Reversing this trend, a decrease appeared from 2001 onward, reaching a reduction of 50\% in 2002. The results showed an increase in API values from 1970 to 2000 , consistent with the incidence profile for malaria occurrence in the Amazon. A reduction in API was observed by 2002, reaching the goals set by the current control program.
\end{abstract}

Malaria; Communicable Disease Control; Incidence
Rosilene Ferreira Cardoso 1,2

Paulete Goldenberg 3,4

\section{Introdução}

Constituindo um antigo problema de saúde pública no mundo, a malária se encontrava na entrada do século XX disseminada por quase todo o território brasileiro. A criação do Instituto Soroterápico Federal no Rio de Janeiro - que daria origem ao Instituto Oswaldo Cruz, em 1908 - e do Instituto Butantã em São Paulo, marcariam um movimento institucional de reorganização dos serviços de higiene, oficializando propostas de enfrentamento das endemias 1 . Na década de 20 , é aprovado regulamento do Departamento $\mathrm{Na}$ cional de Saúde Pública do Ministério da Justiça e Negócios Interiores que estabelecia medidas para profilaxia e controle do impaludismo, ancorados no diagnóstico parasitológico e determinação de índices endêmicos, medidas antilarvárias e tratamento de doentes, ao lado da investigação epidemiológica 2 .

Conferências internacionais promovidas pela Organização Pan-Americana da Saúde (OPAS) ${ }^{2}$ no pós-guerra, insistiam na premência da adoção de políticas abrangentes em âmbito nacional, reforçando a concepção de erradicação da doença. Em 1965 é desencadeada a Campanha de Erradicação e Controle da Malária (CEM) que, privilegiando medidas de controle vetorial, foi capaz, segundo Loiola et al. 3, de eliminar a malária em extensas áreas do país, permanecendo sua ocorrência basicamente restrita à Amazônia. 
Diante da política de ocupação e desenvolvimento da Amazônia 3,4, associada ao afluxo de migrantes a partir dos anos 70, registra-se, a propósito, um quadro de aumento da incidência da malária, paralelamente ao seu recrudescimento no mundo. De 52.469 casos observados na entrada dos anos 70, o país viu crescer este número para 169.871 nos anos 80 . Na seqüência dessas constatações, a CEM é desativada e a coordenação das atividades de controle em âmbito nacional passa para a esfera da Superintendência de Campanhas de Saúde Pública (SUCAM), inaugurando um percurso de mudanças institucionais e de medidas de intervenção 3,4 .

Em meio às dificuldades de atingir toda a região amazônica, é adotada a proposta de estratificação epidemiológica, ancorada na identificação das áreas de risco. Constatada a manutenção da doença, entretanto, questiona-se a concepção de erradicação, bem como a centralização das políticas de controle da doença.

Desde a IV Reunião de Ministros da Saúde das Américas em 1977, a OPAS 5 preconiza a incorporação de cuidados de atenção primária, redirecionando as propostas de intervenção. A implementação dessas diretrizes no país seria perpassada, à entrada dos anos 90, pela instalação do Sistema Único de Saúde (SUS), envolvendo o complexo processo de descentralização, subjacente à implantação da assistência primária 1 .

Diante do quadro de aumento da ocorrência da malária, o Ministério da Saúde recorre ao financiamento do Banco Internacional de Reconstrução e Desenvolvimento (BIRD) para o Projeto de Controle da Malária na Bacia Amazônica (PCMAM), com vigência prevista para o período de 1989 a 1993. Segundo Loiola et al. 3, a reestruturação institucional no âmbito federal, envolvendo a incorporação da SUCAM - responsável pelo PCMAM - na Fundação Nacional de Saúde (FUNASA), de um lado, e dos cortes orçamentários, de outro, acabaram paralisando o projeto. Apesar dos limites apontados, no dizer dos autores 3 , o projeto contribuiu para a estruturação dos serviços locais de saúde, tendo resultado numa importante redução das taxas de mortalidade, porém, não alcançou estimular as estruturas locais, no sentido de capacitá-las a assumir as ações de controle da malária.

A incidência da doença na Amazônia volta a subir no período de 1995-1998 observando-se, de acordo com os referidos autores, um aumento de $26 \%$ do número de casos, ressaltando-se, ao lado do aumento de infecções por Plasmodium falciparum, a elevação do número de óbitos.

Em vista da convocação da OPAS para redução da ocorrência de malária no país, responsável por $40 \%$ dos casos das Américas, reorgani- za-se a proposta de controle da doença que se consubstancia no Plano de Intensificação das Áreas de Controle da Malária na Amazônia Legal (PIACM), registrando-se o compromisso de reduzir em $50 \%$ a incidência da doença no país 6 .

Vale dizer, de acordo com Barata ${ }^{4}$, que as condições epidemiológicas não são homogêneas na Amazônia. Se os seringais, áreas de pastagem, assim como acampamentos de construtoras e garimpos fechados apresentam, em geral, baixa incidência da doença, os garimpos abertos, os assentamentos espontâneos e zonas novas de colonização têm incidência altíssima, em função das atividades de desmatamento e da concentração de suscetíveis entre recém-chegados, quase sempre não-imunes. Diante dessa diversidade de injunções epidemiológicas associadas à intensidade e extensão que a malária pode assumir e, do variante quadro de orientações relacionado com o controle da doença no âmbito da Amazônia, coloca-se a preocupação com a ocorrência da malária no Estado do Amapá.

Ressaltando-se a intensidade do desenvolvimento regional, na situação político-administrativa de um Estado em recente estruturação, a recomposição da trajetória da doença, neste espaço, constitui contexto de referência para o monitoramento da doença. Nesse sentido se inscreve o propósito de dimensionar a incidência da malária no Estado do Amapá, no período de 1970 a 2003.

\section{Metodologia}

Tendo em vista caracterizar a ocorrência e evolução da malária no Estado do Amapá, recorreremos à construção de uma série temporal do índice parasitário anual (IPA). Constituindo medida de incidência da doença, de acordo com o Ministério da Saúde 7, ela expressa o número de lâminas positivas para malária, por mil habitantes.

Levando em conta a carência de informações no estado, o dado de 1970 foi o mais antigo disponível. Vale notar que essa data comporta uma dupla demarcação histórica, de um lado, em função da reorganização das políticas relativas aos Territórios Federais em 1969 8, que coincide com o desencadeamento da transição no sentido da institucionalização do Estado do Amapá. De outro, em função da desativação da CEM, sinalizando para a reorganização dos programas e estruturas de enfrentamento da endemia em nosso meio.

A positividade da infecção resultou da notificação de casos, determinados pela identificação e quantificação do parasito por gota es- 
pessa, conforme procedimentos adotados pelos programas de controle da malária 7 . Ao lado da especificação do tipo de plasmódio (falciparum, vivax, malariae e ovale), ressalta-se que no cálculo do IPA são excluídas as lâminas repetidas por paciente, sendo computados somente os casos notificados.

Os dados de lâminas positivas, de 1970 a 1995 foram obtidos junto à FUNASA, no ano de 1996 a informação foi obtida na Secretaria de Vigilância em Saúde do Ministério da Saúde, e, a partir de 1997, foram computados os dados disponibilizados pelo Sistema de Informação de Malária (SISMAL) e Sistema de Informações de Vigilância Epidemiológica e Notificação de Casos (SIVEP). A propósito da diversidade de fontes, os dados foram verificados tendo em vista a sua consolidação para efeito do presente estudo. As informações sobre população, por sua vez, foram obtidas junto ao Instituto Brasileiro de Geografia e Estatística (IBGE) 9.

Os valores do IPA, estimando o risco de ocorrência anual de casos, foram classificados de acordo com a Organização Mundial da Saúde (OMS), em graus: de baixo risco, os valores inferiores a dez lâminas positivas para cada mil habitantes; de médio risco, os valores entre $10 \%$ o a $49,9 \%$; e, de alto risco, valores iguais ou superiores a $50 \%$ o 7 .

\section{Resultados}

Os dados de lâminas positivas (Tabela 1) evidenciam a presença, no Estado do Amapá, de todas as espécies de plasmódios existentes no país.

De 1970 a 1978 as lâminas positivas de P.falciparum cresceram de 1.104 para valores superiores a 3.500. Após um decréscimo, o número de lâminas positivas volta a subir até 1989 , atingindo o valor de 4.896. Ampliando os patamares de sua presença após ter alcançado valores similares ao do início da série, estes números voltam a subir, chegando à casa dos 6 mil a partir de 2001. No caso da infecção por vivax, o crescimento de lâminas positivas é ascendente até 1993, atingindo valores superiores a 10 mil; após 1994, este valor atinge a casa dos $30 \mathrm{mil} \mathrm{no}$ ano 2000. A Figura 1 expressa, visualmente, o comportamento diferencial dessas espécies no período.

Considerando, por sua vez, a população do estado, observa-se um aumento progressivo (Tabela 1) evidenciando um crescimento de 4,6 vezes a população de 1970 (que era de 116 mil habitantes). A Figura 2 permite visualizar o crescimento acelerado da população do estado, sendo possível identificar pontos de inflexão significativos. Em 1978 o crescimento, em relação a 1970, havia sido de $146 \%$; esta proporção aumenta para 232\% em 1990, atinge $281 \%$ em 1996 e 461\% em 2003. Destaca-se, a propósito, o incremento de $59 \%$ no intervalo de 1978 a 1990 e, de $64 \%$ no período de 1996 a 2003.

Calculado o IPA (Tabela 1), observamos um aumento desses valores no conjunto do período, ou seja, de 1970 a 2003. Ressaltando as oscilações constitutivas do comportamento do referido índice no decorrer desses anos, conforme evidencia a Figura 3, observamos um pico em 1971 (IPA de 37,4\%o) seguido de nova elevação em 1976, quando o IPA atinge o valor de 52,8 casos positivos por mil habitantes. Na seqüência de um movimento descendente até 1980, estruturam-se, entretanto, dois novos picos: um deles com um IPA de 39,6\%o em 1990 e, outro, no ano 2000, com um IPA de $74 \%$ - renovando uma situação de alto risco, só observada anteriormente em 1977.

Finalizando a série, os dados apontaram para uma redução da ordem de $50 \%$ dos valores do IPA, em 2002. Mantido o mesmo nível em 2003, ressalta-se que esse valor corresponde ao dobro do IPA de 1970.

\section{Discussão}

O registro da elevação da malária desde os anos 70 acompanha o comportamento de aumento relatado para a Amazônia no período 3,4,6, reiterando a semelhança de situações de risco associadas às formas e intensidade da ocupação empreendidas pelo homem. Tendo alcançado o valor máximo em 2000 - demarcando a evolução de uma situação de médio para alto risco - os dados revelam uma queda nos anos subseqüentes, que se mantém, entretanto, em níveis superiores aos de 1970.

Num movimento de ascensão não-linear, este percurso se faz marcado por oscilações que se estruturam, certamente, na dependência dos programas de combate à doença contingenciado pela própria dinâmica de renovação das injunções epidemiológicas regionais.

No início da série, o pico de 1971 ocorre na vigência da atuação da SUCAM, a qual, sem a mesma estrutura da CEM, não dá conta de atender, no dizer de Loiola et al. ${ }^{3}$, os contingentes populacionais que afluíam no contexto da política de desenvolvimento da Amazônia, atraídos pelos grandes projetos regionais. Conforme o jornal Amapá Informativo 10, nesse contexto se inscreveria o aumento da malária no Estado, explicitando que o Amapá não fugia à regra, embora com menor número de casos em relação aos demais Estados da Amazônia. 
Lâminas positivas de malária por espécie de plasmódio, população e índice parasitário anual - IPA (por mil habitantes).

Estado do Amapá, Brasil, 1970 a 2003.

\begin{tabular}{|c|c|c|c|c|c|c|c|}
\hline \multirow[t]{2}{*}{ Ano } & \multicolumn{5}{|c|}{ Lâminas positivas * } & \multirow[t]{2}{*}{ População ** } & \multirow[t]{2}{*}{ IPA } \\
\hline & P. falciparum & P. vivax & $P$. falciparum $+P$. vivax & P. malariae & Total & & \\
\hline 1970 & 1.104 & 650 & 3 & 1 & 1.758 & 116.000 & 15,1 \\
\hline 1971 & 2.990 & 1.338 & 6 & 2 & 4.363 & 116.000 & 37,4 \\
\hline 1972 & 1.603 & 1.018 & 15 & 0 & 2.636 & 130.102 & 20,3 \\
\hline 1973 & 592 & 682 & 5 & 0 & 1279 & 135.000 & 9,5 \\
\hline 1974 & 1.652 & 1.443 & 17 & 0 & 3.112 & 137.000 & 22,7 \\
\hline 1975 & 3.529 & 2.357 & 53 & 1 & 5.940 & 132.066 & 45,0 \\
\hline 1976 & 3.683 & 3.282 & 65 & 0 & 7.030 & 133.131 & 52,8 \\
\hline 1977 & 3.540 & 2.919 & 44 & 1 & 6.504 & 140.229 & 46,4 \\
\hline 1978 & 3.864 & 2.911 & 42 & 1 & 6.818 & 169.779 & 40,1 \\
\hline 1979 & 1.996 & 1.708 & 21 & 3 & 3.728 & 183.935 & 20,3 \\
\hline 1980 & 611 & 1.106 & 10 & 0 & 1.729 & 184.853 & 9,3 \\
\hline 1981 & 1.454 & 1.347 & 11 & 0 & 2.812 & 199.477 & 14,1 \\
\hline 1982 & 2.322 & 2.800 & 17 & 0 & 5.139 & 214.309 & 24,0 \\
\hline 1983 & 2.154 & 2.485 & 15 & 0 & 4.654 & 213.664 & 21,8 \\
\hline 1984 & 3.071 & 4.164 & 25 & 0 & 7.260 & 244.720 & 29,7 \\
\hline 1985 & 3.468 & 3.969 & 24 & 0 & 7.461 & 250.046 & 29,8 \\
\hline 1986 & 3.849 & 4.330 & 40 & 0 & 8.219 & 265.498 & 31,0 \\
\hline 1987 & 3.117 & 4.272 & 89 & 0 & 7.478 & 274.248 & 27,3 \\
\hline 1988 & 4.316 & 5.198 & 61 & 0 & 9.575 & 293.773 & 32,6 \\
\hline 1989 & 4.896 & 6.190 & 70 & 0 & 11.156 & 295.583 & 37,7 \\
\hline 1990 & 4.362 & 6.272 & 43 & 0 & 10.677 & 269.633 & 39,6 \\
\hline 1991 & 3.411 & 5.342 & 37 & 3 & 8.793 & 289.387 & 30,4 \\
\hline 1992 & 2.379 & 4.558 & 30 & 2 & 6.969 & 299.305 & 23,3 \\
\hline 1993 & 1.276 & 3.715 & 22 & 7 & 5.020 & 308.783 & 16,3 \\
\hline 1994 & 1.778 & 10.450 & 21 & 5 & 12.254 & 317.597 & 38,6 \\
\hline 1995 & 2.650 & 14.326 & 51 & 4 & 17.031 & 326.188 & 52,2 \\
\hline 1996 & 2.311 & 16.695 & 32 & 18 & 19.056 & 379.459 & 50,2 \\
\hline 1997 & 1.958 & 22.863 & 41 & 6 & 24.868 & 401.916 & 61,8 \\
\hline 1998 & 2.216 & 20.312 & 33 & 8 & 22569 & 420.834 & 53,6 \\
\hline 1999 & 3.050 & 25.552 & 43 & 1 & 28.546 & 439.781 & 65,1 \\
\hline 2000 & 5.108 & 30.074 & 92 & 4 & 35.278 & 477.032 & 74,0 \\
\hline 2001 & 6.289 & 18.075 & 104 & 19 & 24.487 & 498.735 & 49,1 \\
\hline 2002 & 4.812 & 11.060 & 83 & 302 & 16.257 & 516.511 & 31,5 \\
\hline 2003 & 6.027 & 10.309 & 218 & 87 & 16.643 & 534.835 & 31,1 \\
\hline
\end{tabular}

* Fonte: Sistema de Informações de Vigilância Epidemiológica-Malária, Fundação Nacional de Saúde/Secretaria de Vigilância em Saúde, Ministério da Saúde.

** Fonte: Instituto Brasileiro de Geografia e Estatística.

Num quadro de crescimento populacional relativamente reduzido, por referência ao período focalizado, o aumento observado corresponderia, segundo dados disponibilizados pelo Ministério da Saúde 2, a um momento onde o combate à malária se assentaria na borrifação intradomiciliar, realizada sem regularidade. Acrescentar-se-iam, ainda, problemas de ordem operacionais diante das migrações, nomadismo regional, dispersão, assim como a questões re- lativas à densidade e outras características do vetor, ressaltando-se, particularmente, o registro da presença de cepas de P. falciparum resistentes à cloroquina.

Diante do aumento observado, se estabelecem medidas regulares, o que poderia explicar a queda subseqüente até 1973, quando os valores do IPA atingem os níveis mais baixos do período, inferiores a 1970 . 
Figura 1

Lâminas positivas de malária, por espécie de plasmódio (falciparum e vivax). Estado do Amapá, Brasil, 1970 a 2003.

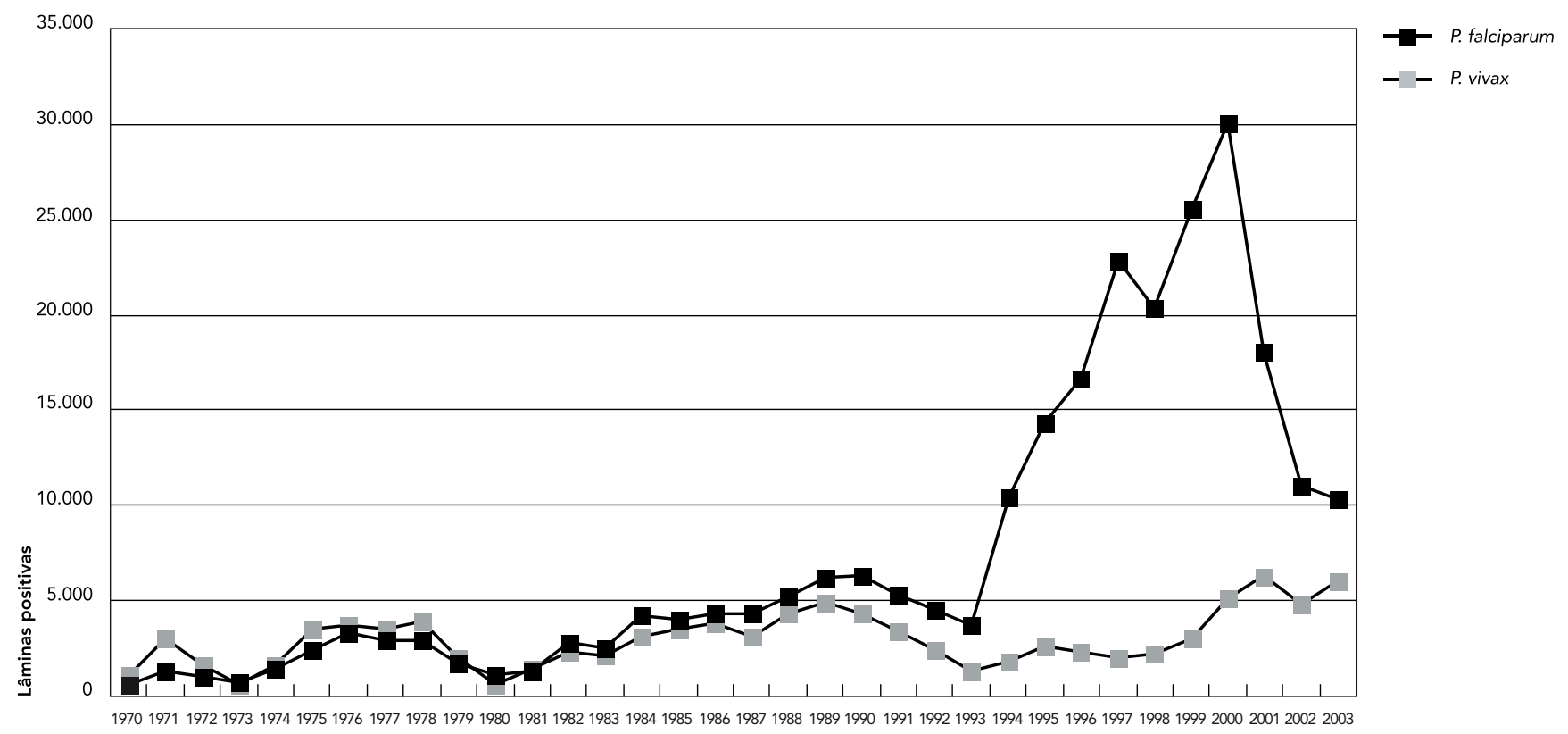

Fonte: Sistema de Informações de Vigilância Epidemiológica-Malária, Fundação Nacional de Saúde/Secretaria de Vigilância em Saúde, Ministério da Saúde.

Figura 2

População do Estado do Amapá, Brasil, 1970 a 2003.

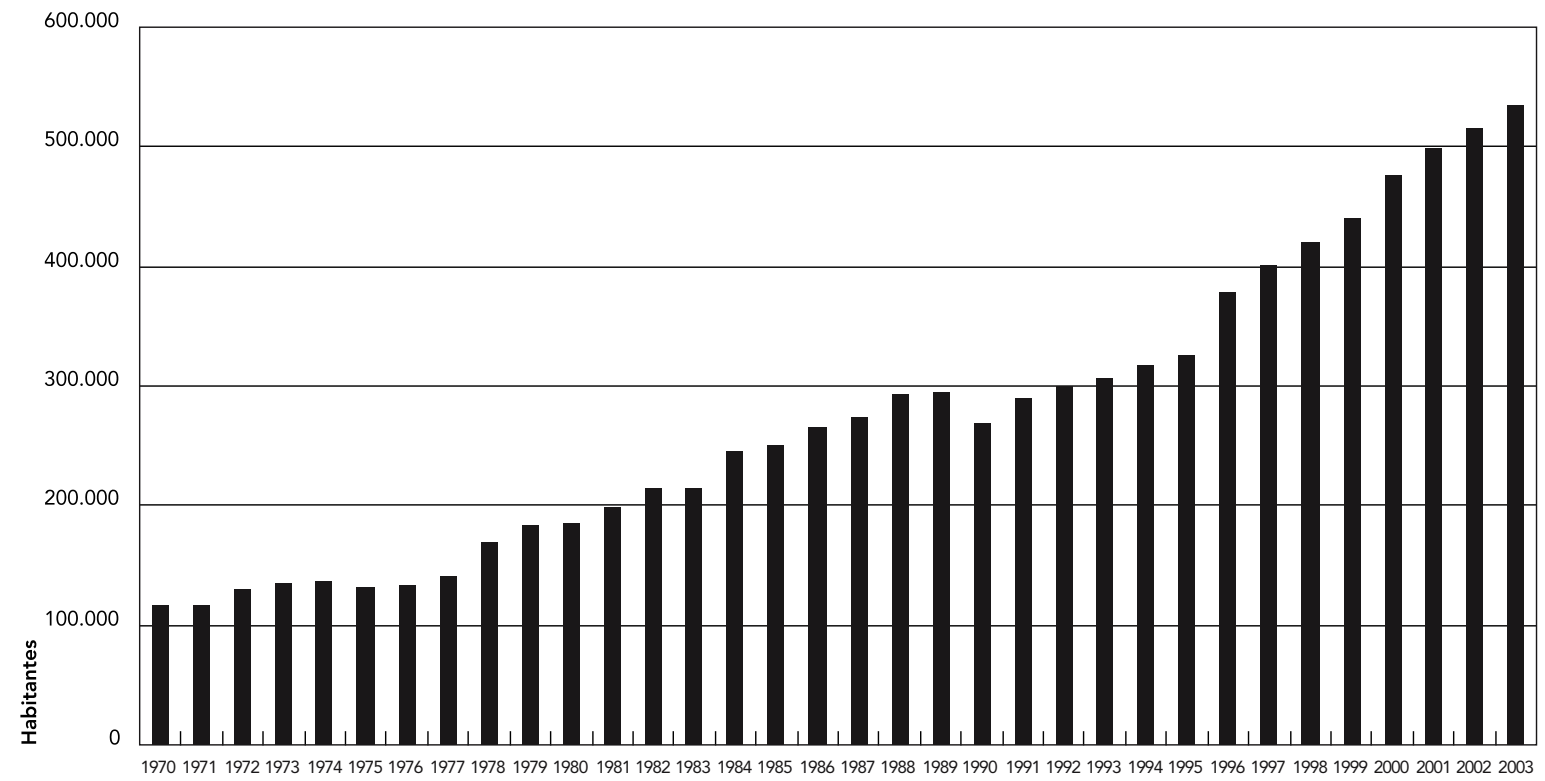

Fonte: Instituto Brasileiro de Geografia e Estatística. 


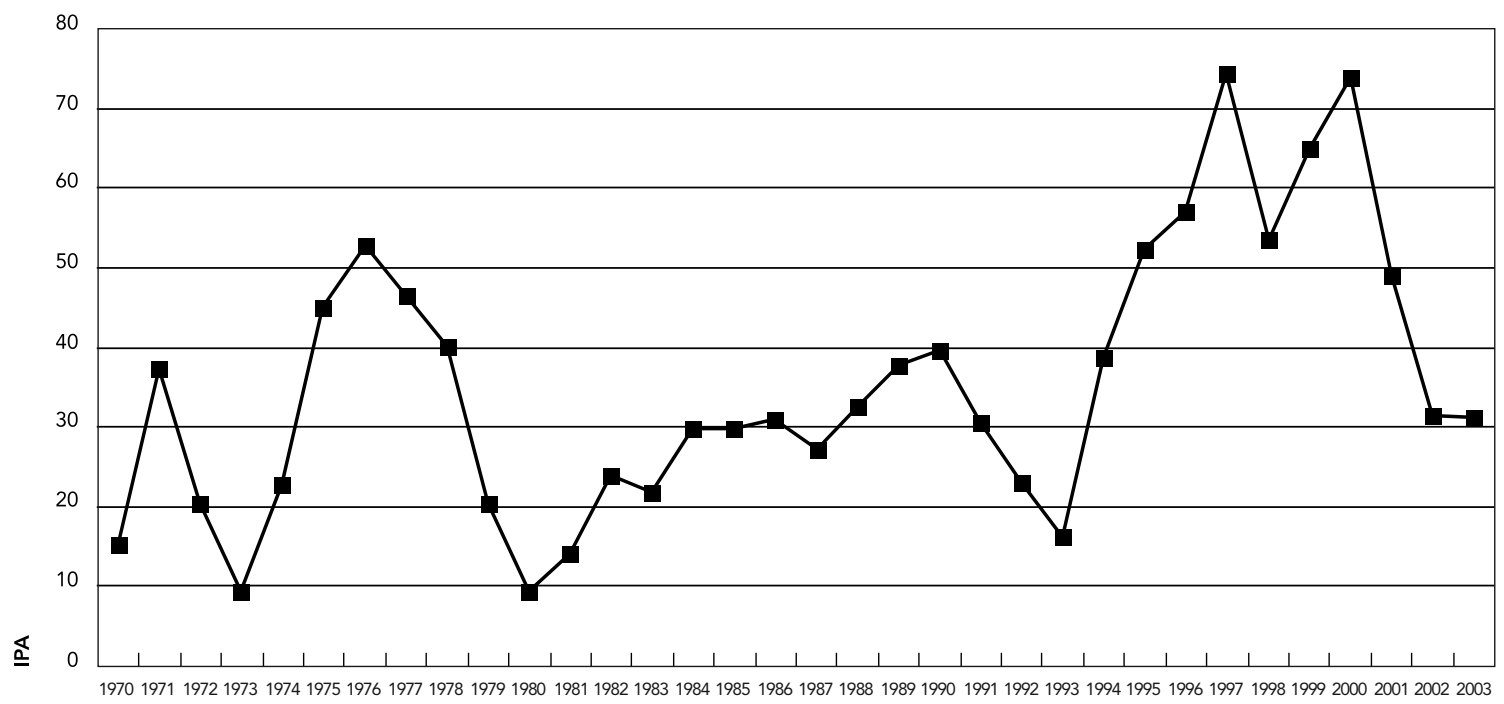

Fonte: Sistema de Informações de Vigilância Epidemiológica-Malária, Fundação Nacional de Saúde/Secretaria de Vigilância em Saúde, Ministério da Saúde/ Instituto Brasileiro de Geografia e Estatística.

A partir de 1974, porém, a malária volta a subir, constituindo um elevado pico em 1976, que coincide com o aumento de positividade para falciparum (as lâminas positivas passaram de 592, em 1973, para 3.529 em 1975, mantendo estes níveis até 1978). Registra-se, nesse período, a inauguração da hidroelétrica de Paredão em 1976 8, a qual daria suporte ao desenvolvimento econômico regional; a instalação do Projeto Jari, ao lado da fábrica de celulose. A composição do parque industrial em desenvolvimento demarcaria, ao lado do desmatamento, o afluxo de migrantes e da conseqüente concentração de suscetíveis entre os recém-chegados, configurando o ambiente favorável à transmissão da doença.

$\mathrm{Na}$ ausência de dados específicos da literatura que explicassem a queda subseqüente, não se poderia deixar de considerar a mobilização de forças-tarefas diante da sinalização de alerta, ressaltando-se, com certa defasagem, a diminuição de lâminas positivas para falciparum (que voltam para 611 em 1980). A propósito, acrescentam-se observações do I Encontro de Malária no Estado (na época ainda Território) que referiam a adaptação de medidas de controle de borrifação em função do material utilizado na construção das casas, que limitava a aderência do dicloro difenil tricloretano (DDT), ressaltando-se a borrifação do assoalho da habitação e a periodicidade da aplicação, que passava a ser feita a cada três meses em vez de seis meses 11 .

Nesse movimento descendente que sucede ao pico de 1976, registra-se a introdução pela SUCAM no início dos anos 80 , do recurso às estratégias de estratificação epidemiológica que, identificando áreas de risco, procede a adaptações do controle da malária às condições locais de recursos financeiros e humanos ${ }^{3}$. A esta política corresponde uma queda do IPA, acentuando tendência em curso, que alcança os mesmos níveis de 1973. Porém, diante das dificuldades de sustentação de tal estratégia, os valores do IPA voltariam a subir.

Sem pretender uma relação direta entre crescimento da população e os níveis de comportamento do IPA no Estado, o crescimento populacional, desde 1978, apontado como um ponto de inflexão, poderia ser considerado como um dos fatores que teriam solapado as medidas apoiadas na estratificação epidemiológica.

A partir de 1981 configura-se um novo movimento ascendente dos valores do IPA no Amapá, que se estende até 1990, quando atinge o valor de $39,6 \%$. Se esse valor não diferia muito do ob- 
servado em 1971, chama atenção a duração da tendência desse aumento. Esse movimento se faz acompanhar de um significativo aumento de positividade por $P$. falciparum, que passa, conforme referência anterior, de 611 lâminas positivas, em 1980, para 4.896 em 1990, acrescido do aumento de P. vivax, que passaria, nos respectivos anos, de 1.106 lâminas positivas para 6.272, demarcando, a partir daí, a supremacia de sua presença sobre o P. falciparum.

Diante do início do novo patamar de aumento populacional observado em 1978, ressalta-se, a propósito, a destacada proporção de crescimento, da ordem de 59\%, até 1990 , cuja população alcançaria 232\% em relação ao valor de 1970 .

Com base no relato do I Encontro, a ascensão da malária, no referido período, coincidiria com a introdução do garimpo de ouro de aluvião ${ }^{11}$. De um núcleo isolado no Lourenço, no Município de Calçoene, eles passam, no período de 1980 a 1984, de 5 para 20 naquela localidade; de 1 para 5 no Araguari; e, de 4 para 32 em Amapari. Somando 57 unidades, com uma população estimada de 12 mil garimpeiros, avaliações epidemiológicas da época revelavam que os garimpos respondiam por $79 \%$ do total de casos de malária do estado.

Barata 4 distingue condições diferenciais de risco entre o garimpo aberto e fechado - reforçadas pelos achados de Couto et al. 12, que relatariam a implantação de programas de controle da endemia em áreas de garimpo do Lourenço. Apesar disso, seria de se esperar que, na situação de explosão desses garimpos, prevalecessem assentamentos pioneiros, com núcleos urbanos embrionários, os quais albergariam injunções favoráveis à transmissão da malária. Diante do afluxo de migrantes, quase sempre não-imunes, a infecção desencadeia quadros agudos, principalmente entre adultos envolvidos nas frentes de trabalho. Os nativos, entretanto, expostos à infecção desde cedo, desenvolvem imunidade em algum grau, permanecendo assintomáticos, fora do alcance de medidas de controle 13,14.

No plano da organização das políticas de saúde, em 1988 ocorre a institucionalização do SUS, envolvendo a instalação de complexo processo de descentralização, cuja implantação constituiria um desafio a ser perseguido. Tais desdobramentos esbarrariam com o cenário de institucionalização do Estado em curso, dando cumprimento, no plano político-administrativo, às leis orgânicas dos territórios, que se concretizariam, em 1985, com as primeiras eleições de prefeitos e, do primeiro governador do Estado em 1990. Em meio a esses desdobramentos, diante de um quadro cada vez mais grave de crescimento da malária na região, o governo celebra, em 1989, um convênio com o BIRD, de apoio financeiro à instalação do PCMAM no espaço da Bacia Amazônica, às vésperas do pico de 1990. Em meio à paralisação decorrente dos rearranjos institucionais em âmbito federal, o programa só é ativado, efetivamente, em 1993

Conferências internacionais, desde 1992, reiterando estratégia mundial de luta contra o paludismo, acentuavam medidas voltadas para o doente, tendo em vista reduzir a morbidade, impedir a mortalidade, considerando o fortalecimento das ações de locais de saúde. Como desdobramento dessas disposições, é elaborado o Programa de Controle Integrado da Malária (PCIM), com ênfase no diagnóstico e tratamento dos doentes, detecção precoce de epidemias e aplicação seletiva de controle de vetores etc. - que não chega a ser completamente instalado ${ }^{3}$. No quadro de aumento da doença a partir de 1995, de acordo com os mesmos autores, se estabelece o Plano de Intensificação das Ações de Controle da Malária nas Áreas de Alto Risco, que ensaia uma aproximação junto aos municípios, com limitado sucesso.

Tecendo um balanço dessas iniciativas, entretanto, Loiola et al. 3 consideram que esses planos resultaram em ganhos importantes. O programa havia avançado na instalação da rede de laboratórios para diagnóstico e tratamento, ao lado da capacitação de recursos humanos, propiciando significativa redução das taxas de mortalidade na região. Reconhecendo a diminuição da incidência de $P$. falciparum, os autores assinalam que, a despeito da maior racionalidade das ações, o controle direcionado à concentração de vetores acabou perdendo força na maioria dos Estados. Finalmente, a participação incipiente dos municípios teria apontado para a dificuldade de desenvolver estruturas locais. Ainda na dependência dos órgãos centrais, diante da retração dos investimentos governamentais de 1995 a 1998, observa-se novo recrudescimento da endemia na Amazônia, reiterando-se as dificuldades de efetivar o processo de descentralização.

Os valores do IPA no Estado do Amapá continuam a subir alcançando o maior pico da série no ano 2000. Esses dados coincidem, sugestivamente, com o aumento da malária verificado no Pará 15, no período de 1989 a 1998, destacando-se a relevância de sua presença no âmbito na Amazônia Legal e a contigüidade com o Estado do Amapá.

Numa aproximação ao cenário desse aumento, vale registrar a criação da Área de Livre Comércio, em 1991, que renovaria a vocação do estado como pólo migratório na Amazônia, ressaltando-se, segundo o IBGE 9, que mais da metade do crescimento populacional do Estado era 
devido à imigração proveniente, principalmente, do Pará, Maranhão e Ceará. Se, o período de 1991 a 1995 havia apresentado uma das menores proporções de crescimento no conjunto da série histórica, da ordem de 13,6\% - o que pode ter contribuído para potencializar os efeitos positivos do PCMAM de 1990 a 1993 - o mesmo não se pode dizer em relação aos anos subseqüentes. A partir de 1996 - tido como um ponto destacado de inflexão do crescimento populacional no intervalo da série histórica - observou-se um aumento significativo da população do estado até o fim do período, da ordem de $64 \%$, sendo que a população do estado em 2003 comportaria um crescimento de $461 \%$ em relação à população de 1970 .

Considerando o período de 1991 a 2000, o IBGE 16 registra que, com exceção da cidade do Amapá, que apresentou uma taxa média geométrica de crescimento anual de $0,76 \%$, e Calçoene, com 2,99\%, todos os demais municípios do estado apresentaram taxa geométrica anual superior a 3\% ao ano, ressaltando-se que em cinco municípios esta taxa variou entre 5,14 e 6,34\%, e, outros cinco municípios, entre 7,21 e 11,99\%. Num movimento de queda de crescimento desde os anos 70, a taxa média geométrica de crescimento anual do país, no mesmo período, foi de $1,64 \%$. A Região Norte, que por sua vez, nos anos 70 havia apresentado uma elevada taxa geométrica de crescimento anual da ordem de 5,02\%, no período de 1991 a 2000 teve esta taxa reduzida para $2,86 \%$.

Atraindo um grande contingente de pessoas oriundas de outros estados, ao lado do aumento da concentração de suscetíveis, pesa o argumento de que o estado emergente não possuía infraestrutura na área de saúde capaz de dar conta de uma população com explosivo crescimento, a despeito do aumento da rede assistencial do estado desde 1976, que passaria de 43 para 221 estabelecimentos em 2002, sendo 184 financiados pela rede SUS, segundo o IBGE 9 .

Criam-se novos bairros na capital Macapá (Brasil Novo, Infraero I e II, Jardim Felicidade I e II, Renascer e Mar Abaixo etc.) e áreas de invasão, locais onde até hoje se mantêm como espaços de alta transmissão, ao lado das extensas áreas de ressaca, propícias à existência de focos de vetores. Soma-se a esses aumentos do volume de casos, a chegada de ribeirinhos inclusive do Estado do Pará que, juntamente com doentes de outros municípios, afluem para os centros de assistência do Estado em busca de tratamento de maior complexidade para malária e para outras doenças - não somente sobrecarregando os serviços existentes, mas colaborando para a transmissão da doença em área urbana.
Após a divulgação da avaliação da situação epidemiológica da malária nas Américas pela OPAS 2, em 1998, justificando a necessidade imediata de encontrar estratégias para manter a doença sob controle, o Brasil apresenta "ousada" proposta de intervenção na reunião internacional em Lima, no Peru, em 1999, afirmando o empenho de reduzir o número de casos de malária em $50 \%$ até o final de 2001 3,6. O PIACM, com base nas experiências anteriores, traz como diferencial, agora, o compromisso político de controle da malária assumido pelas diversas instâncias governamentais (federal, estadual e municipal), respaldada pela visão do custo social da doença e de sua relação com o desenvolvimento regional, ao lado da garantia de um financiamento continuado - certificando, no contexto do SUS, o fim dos programas verticais. Data dessa iniciativa o desencadeamento do movimento descendente do IPA após o pico do ano 2000 - a despeito do continuado aumento populacional.

De acordo com Tauil 6, o PIACM só foi realmente implantado no Estado no segundo semestre de 2001, apresentando, em sua avaliação, uma redução da incidência da malária na ordem de $30,6 \%$, neste mesmo ano. Na vigência do programa, os registros da presente série temporal indicaram uma redução de 35.278 casos em 2000 para 16.257 em 2002. Cumprindo a meta estabelecida de redução da ordem de 50\%, o IPA que era de 74\%o em 2000 cai para 31,5\%o em 2002.

Num quadro de ascensão da notificação por P. falciparum e do crescimento populacional do estado até o fim da série, a queda do IPA se faz sentir envolvendo significativa redução da positividade por $P$. vivax, que cai para um terço dos 30 mil notificados em 2000 , potencializada pelas relações inter e intra-setorial, expressando a aderência ao PIACM.

Vale dizer que em 2001 são definidas as ações integradas entre a FUNASA e Instituto Nacional de Colonização e Reforma Agrária (INCRA) para a implementação do controle da malária nos assentamentos, por meio da portaria interministerial do Ministério da Saúde e Ministério de Desenvolvimento Agrário 17. Ressalta-se, ainda, no setor da saúde, a atuação do Programa Saúde da Família (PSF) e do Programa de Agentes Comunitários de Saúde (PACS) que incorporaram ações de vigilância, prevenção e controle da malária e dengue, conforme Portaria $n^{o} .44$ do Ministério da Saúde 18 - que coincide com a substituição do SISMAL pelo Sistema de Informações de Vigilância Epidemiológica (SIVEP-Malária), ancorando o processo de implantação da assistência primária no âmbito da descentralização. 


\section{Considerações finais}

Observando o conjunto da série histórica, ressalta aos olhos o crescimento da malária no período, registrando-se dois picos seqüenciais que ocorrem no início dos anos 70, por ocasião do desencadeamento da política de ocupação da Amazônia; segue a tendência significativa do aumento associado à proliferação do garimpo na região e, finalmente, a constituição de período explosivo de crescimento dos valores do IPA que segue a instalação da Área de Livre Comércio.

Mobilizando ações de controle, evidencia-se no decorrer desses esforços uma ação pendular que passa da ênfase da redução do índice vetorial para o atendimento ao doente. Expressando a vontade política, fundamentada no conhecimento disponível, essas medidas resultam em inflexões descendentes - até o limite de sua capacidade em atender à renovação das condições favoráveis à transmissão da malária, das quais o homem é um dos protagonistas. A propósito, não se pode perder de vista as considerações de Barata 4 sobre as orientações apoiadas pelas conferências internacionais, alertando para o perigo de ver superdimensionadas a atenção ao doente em detrimento da doença.

Sem pretender esquadrinhar o complexo quadro epidemiológico subjacente à ocorrência da malária no âmbito regional, vale registrar que estudos recentes detectaram grande número de portadores de $P$. vivax e $P$ falciparum em populações ribeirinhas, entre indivíduos assintomáticos. Comprovando a hipótese da capacidade desses portadores infectarem os mosquitos ao longo da vida, compensando os baixos níveis de parasitemia, os autores concluem que eles atuam como reservatório passivo da doença, constituindo, assim, fonte permanente de manutenção do ciclo de transmissão para os migrantes, nãoimunes 13,14.

Tais achados enfatizam a necessidade de se desenvolver estratégias inovadoras para o controle da malária, considerando a realização de diagnóstico e tratamento de portadores assintomáticos. Diante da inoperância das medidas convencionais voltadas para os vetores domiciliados, baseadas na aplicação de inseticidas nessas populações ribeirinhas, a única medida factível, segundo os referidos autores, é o tratamento dos assintomáticos, como recurso para atingir o reservatório de plasmódios.

Pensar a doença numa dimensão abrangente, revendo a articulação do plano biológico e social, constitui, certamente, um requisito fundamental no sentido de aprofundar os avanços envidados no enfrentamento da doença em âmbito regional.

\section{Resumo}

O presente estudo tem por objetivo dimensionar a ocorrência de malária no Estado do Amapá, Brasil, no período de 1970 a 2003. Com dados de lâminas positivas, obtidos junto a agências de informações em saúde, foi construída uma série temporal de índice parasitário anual (IPA), por mil habitantes, considerando as informações de população obtidas junto ao Instituto Brasileiro de Geografia e Estatística. No contexto de um intenso incremento populacional, associado ao desenvolvimento regional, os resultados evidenciaram um aumento no período - não linear - dos valores de IPA, que passaram de 15,1\%o, em 1970, para $74 \%$ no ano 2000. No reverso desse movimento, em meio aos esforços de controle da doença, observa-se uma queda desses valores a partir de 2001, que alcança a ordem prevista de 50\% em 2002. Acompanhando o movimento de incidência parasitária anual da malária na Amazônia, esses resultados apontaram para o aumento dos valores do IPA no período de 1970 a 2000, observando-se uma redução nos anos subseqüentes, cumprindo com as metas estabelecidas sob o programa vigente de controle da malária no Estado.

Malária; Controle de Doenças Transmissíveis; Incidência

\section{Colaboradores}

R. F. Cardoso foi responsável pelo levantamento e tabulação dos dados. Ambas as autoras procederam à revisão da literatura, sendo que P. Goldenberg foi responsável pela redação do artigo. 


\section{Referências}

1. Lima NT, Fonseca CMO, Hochman G. A saúde na constituição do Estado Nacional no Brasil: reforma sanitária em perspectiva histórica. In: Lima NT, Guershman S, Fonseca CMO, Hochman G, organizadores. Saúde e democracia: historia e perspectivas do SUS. Rio de Janeiro: Editora Fiocruz; 2005. p. 27-58.

2. Organização Pan-Americana da Saúde/Organização Mundial da Saúde. Trajetória de um sanitarista - recompilação da produção intelectual do Dr. Agostinho Cruz Marques. Brasília: Organização Pan-Americana da Saúde/Organização Mundial da Saúde; 1998.

3. Loiola CC, Silva CJM, Tauil PL. Controle da malária no Brasil: 1965 a 2001. Rev Panam Salud Pública 2002; 11:235-43.

4. Barata RCB. Malária no Brasil: panorama epidemiológico na última década. Cad Saúde Pública 1995; 11:128-36.

5. Organización Panamericana de la Salud/Organización Mundial de la Salud. Extensión de cobertura de servicios de salud con las estrategias de atención primaria y participación de la comunidad. Washington DC: Organización Panamericana de la Salud/Organización Mundial de la Salud; 1978. (Documento Oficial, 155).

6. Tauil PL. Avaliação de uma nova estratégia de controle da malária na Amazônia Brasileira [Tese de Doutorado]. Brasília: Universidade de Brasília; 2002.

7. Departamento de Informática do SUS. IDB 2000. Informações de saúde. http://www.datasus.gov.br (acessado em 03/Ago/2002).

8. Santos FR. História do Amapá. Da autonomia territorial ao fim do Janarismo - 1943-1970. Amapá: Editora Gráfica O Dia; 1998.

9. Instituto Brasileiro de Geografia e Estatística. Censo demográfico 2000. Rio de Janeiro: Instituto Brasileiro de Geografia e Estatística; 2002.
10. Anônimo. Malária em debate - Macapá. Amapá Informativo 1984; 15 nov.

11. Ministério da Saúde. I Encontro sobre Malária no Território Federal do Amapá. Macapá: Ministério da Saúde; 1984.

12. Couto AA, Calvosa VS, Lacerda R, Castro F, Rosa ES, Nascimento JM. Controle de transmissão da malária em área de garimpo no Estado do Amapá com a participação da iniciativa privada. Cad Saúde Pública 2001; 17:897-907.

13. Alves FP, Durlacher RR, Menezes MJ, Krieger H, Silva LH, Camargo EP. High Prevalence of asymptomatic Plasmodium vivax and Plasmodium falciparum infections in Native Amazonian populations. Am J Trop Med Hyg 2002; 66:641-8.

14. Alves FP, Gil LHS, Marreli MT, Ribolla PEM, Camargo EP, Silva LHP. Assymptomatic carriers of Plasmodium spp. as infection source for malaria vector mosquitoes in Brazilian Amazon. J Med Etnol 2005; 42:777-9.

15. Cordeiro CE, Filomeno CR, Costa CMA, Couto AA. Perfil epidemiológico da malária no Estado do Pará em 1999 com base numa série histórica de dez anos (1989-1999). Inf Epidemiol SUS 2002; 11:6777.

16. Instituto Brasileiro de Geografia e Estatística. Tendências demográficas: uma análise dos resultados da amostra do censo demográfico 2000. Rio de Janeiro: Instituto Brasileiro de Geografia e Estatística; 2004. (Série Estudos e Pesquisas: Informações Demográficas e Sócio-econômicas, 13).

17. Ministério da Saúde/Ministério do Desenvolvimento Agrário. Portaria interministerial no. 279. Diário Oficial da União 2001; 8 mar.

18. Ministério da Saúde. Portaria nº. 44. Diário Oficial da União 2002; 3 jan.

Recebido em 07/Abr/2006

Versão final reapresentada em 01/Dez/2006

Aprovado em 26/Dez/2006 